\title{
Adverse maternal and perinatal outcomes of elective induction of labour at term vs spontaneous onset of labour; a comparison study
}

\author{
Dasanayake DLW' ${ }^{1}$, Ukwaththa UDML ${ }^{2}$, Gamage $\mathbf{M}^{2}$ \\ Department of Obstetrics and Gynaecology ${ }^{1}$, BSc Nursing Degree Programme ${ }^{2}$, Faculty of Medicine, University \\ of Ruhuna, Galle, Sri Lanka.
}

Correspondence: Dr. D L W Dasanayake

e-mail: lanka.dasanayake@yahoo.com

\begin{abstract}
Introduction: Induction of labour (IOL) is a relatively common procedure in the present obstetrics practice. Decision is made by obstetricians when the extra uterine life is more favourable for the baby than intrauterine environment. However there is evidence that IOL may adversely affect both maternal and perinatal health.

Methods: A group of 75 mothers who underwent IOL was compared with an age-matched sample of same number of mothers who underwent spontaneous onset of labour (SOL) at term at Teaching Hospital Mahamodara. Data were collected from bed head tickets. Main maternal and perinatal outcomes were compared using chi-square and t-tests.

Results: There were no statistically significant differences in the basic demographic characteristics of the two groups. Mothers with IOL had significantly high rates of caesarean section $(p=0.011)$ and prolonged hospital stay $(p<0.001)$ compared to SOL group. There were no statistically significant differences in the two groups with regards to duration of labour $(p=0.073)$, APGAR at 10 minutes $(p=0.466)$ and Special Care Baby Unit (SCBU) admissions $(p=0.405)$.

Conclusions: Mothers who underwent elective IOL at term had increased rate of cesarean section and prolonged hospital stay when compared to mothers who had SOL. The two groups were comparable with regards to adverse perinatal and maternal outcomes.
\end{abstract}

Keywords: Induction of labour, spontaneous onset of labour

\author{
Introduction \\ Induction of labour (IOL) is a process of \\ commencing labour artificially by uterine \\ stimulation. This is carried out if the wellbeing or \\ life of mother or child is threatened by continuance \\ of the pregnancy (1). This procedure is relatively \\ common in modern obstetric practice and is on rising \\ trend. Rate of elective IOL varies worldwide being \\ $6 \%$ to $20 \%$ of pregnancies in developed countries \\ like United Kingdom (2). \\ In modern concept of active management of labour, \\ IOL is an essential component. Main indications for
}

IOL are post-date pregnancies (beyond 41 weeks of gestation), pregnancy complicated with medical disorders (gestational diabetes mellitus, pregnancy induced hypertension and connective tissue disorders, etc.) and fetal growth restriction.

Prolonged pregnancy occurs in 5 to $10 \%$ of all women (3). Epidemiological study supports the view that a pregnancy beyond 40 weeks of gestation is associated with increased perinatal risk (4). This may be even higher for south Asian women (5). Compared with expectant management, IOL is associated with fewer perinatal deaths and caesarean 
sections (6). IOL for other obstetric complications are generally done following optimum fetal lung maturity (beyond 37 completed weeks). Success rate of achieving vaginal delivery is dependent on optimal ripening of cervix prior to induction proper.

Maternal and neonatal effects of IOL are unclear with conflicting evidence. There are many studies performed to compare women with IOL to those undergo spontaneous labour (SOL). Unfortunately, labour induction may itself causes problems especially when the cervix is not favourable. Obstetric problems associated with IOL with an unfavourable cervix include caesarean section, prolonged labour, postpartum haemorrhage and traumatic birth. It is likely that some of these unwanted outcomes result from intervening when the uterus and cervix are not ready for labour (7). IOL is widely practiced to try and prevent the problems mentioned above and improve the health for women and their infants (8).

With retrospective evidence indicating that early term delivery confers higher risk for subsequent adverse neonatal and childhood outcomes compared with later term weeks, the American College of Obstetrics and Gynaecology has issued recommendations to reduce nonmedically indicated induction of labour at less than 39 weeks of gestation (9). Late term or post term pregnancy (greater than 41 or 42 completed weeks of gestation) is a common indication for induction. Recent evaluation of strategies to reduce induction in the absence of medical indication before 39 weeks of gestation has reported decrease in admission to the neonatal intensive care unit (NICU), conflicting results about stillbirth and little information about caesarian delivery, historically one of the key concerns surrounding induction without medical indications (10).

Induced labour also has an impact on the birth experience of women. It may be less efficient and is usually more painful than spontaneous labour and epidural analgesia and assisted delivery are more likely to be required (11). Nearly $45 \%$ of women considered that labour was more painful than expected with induction. The limited available evidence suggests that women feel less satisfaction with experience of IOL than SOL (12).
It is evident that maternal and neonatal outcomes are badly affected with the induction of labour without clear justification leading to increased health cost to a country. Overall, this adversely affects the health economy of countries with low resource setting and increased perinatal and maternal morbidity and mortality.

Even though several studies done with this regards in other countries, we have anecdotal evidence pertaining to IOL. Our pregnant population is different, ethnically and geographically, when compared to the rest of the world and the maternal and neonatal outcomes of Sri Lankan obstetric population following IOL are largely unknown. The aim of this study was to determine rate of adverse maternal and perinatal outcomes of elective induction of labour compared to spontaneous onset of labour.

\section{Methods}

This study was carried out as a comparative crosssectional study at Teaching Hospital Mahamodara, Galle from January 2017 to March 2017. We studied pregnant women at term (gestational age between 37 completed weeks to 41 weeks) who had either elective IOL or SOL. Inclusion criteria were pregnant women at term with singleton live pregnancy that had induction of labour or spontaneous labour. Exclusion criteria were maternal sepsis following delivery, pre labour rupture of membrane, fetal growth retardation and fetal anomalies. Mothers were recruited for the study from postnatal wards. Consecutive eligible postnatal mothers were selected with age matched control (similar age in years in both group) subjects who delivered her baby on the same day. Consideration was made to select control group to have gestational age at least within 07 days of study subjects.

Data was obtained from Bed Head Tickets using data sheet. Data sheet was developed to get required variables including basic characteristics, details of labour, maternal and perinatal outcomes. The main maternal outcome variables were caesarean section rate, duration of labour (time duration from admission to labour ward to delivery), maternal hospital stay (time duration from delivery to 
discharge), genital tract trauma and postpartum haemorrhage. The perinatal outcomes were APGAR score at birth, neonatal Special Care Baby Unit (SCBU) admissions and neonatal deaths. Ethical clearance was obtained from the Ethical Review Committee of the Faculty of Medicine, University of Ruhuna. Permission to conduct the study was granted by the Director and relevant consultants at Teaching Hospital Mahamodara, Galle. Written informed consent was obtained from all postnatal women who participated in the study. Statistical Package of Social Science (SPSS) version 20 was used for data analysis and $p<0.05$ was considered statistically significant. Categorical data were compared with the Chi-square test and continuous variables were compared with the t-test.

\section{Results}

Total number of women in the study was 150 with 75 in each group and the two groups were comparable with regard to the basic demographic characteristics (Table 1).

There was significantly higher rate of cesarean section in the IOL group compared with the SOL group (Table 2).
The rate of caesarian section in the IOL group was almost double compared to the SOL group. In contrast vaginal delivery rate was higher in women with SOL than the women with IOL. However, there was no significant difference in the duration of labour between SOL (mean: $177.61 \pm 34.54$ minutes) and IOL (mean: $218.48 \pm 40.03$ minutes) women with vaginal delivery $(p=0.15)$.

There was a significant difference in the number of days of hospital stay between the two groups. The mean duration of hospital stay in the IOL group was almost double when compared to the SOL group (Table 3).

There was no $3^{\text {rd }}$ degree or $4^{\text {th }}$ degree genital tract trauma among women in the two groups. Each group had one woman with postpartum haemorrhage.

There was no significant difference in APGAR score at 10 minutes at birth between the two groups (Table 4).

There was no significant difference between the two groups with regards to the SCBU admission of their babies (Table 5). There was only one neonatal death which was in the IOL group.

Table 1: Basic characteristics of women with SOL and IOL $(n=150)$

\begin{tabular}{lccccc}
\hline Characteristics & \multicolumn{2}{c}{ SOL (75) } & \multicolumn{2}{c}{ IOL (75) } \\
& Mean & SD & Mean & SD & p value \\
\hline Age (years) & 26.6 & 4.8 & 28.7 & 5.0 & 0.058 \\
Gestational age (days) & 278 & 5.7 & 271 & 5.8 & 0.055 \\
BMI & 21.0 & 2.3 & 20.0 & 2.2 & 0.053 \\
\hline
\end{tabular}

Test - independent sample $\mathrm{t}$

Table 2: Cesarean section rate between two groups of SOL and IOL

\begin{tabular}{lllll}
\hline & LSCS & NVD / Instrumental & $\mathbf{X}^{2}$ & $\boldsymbol{p}$ value \\
\hline SOL & 14 & 61 & & \\
& $18.67 \%$ & $81.3 \%$ & 6.481 & 0.011 \\
IOL & 28 & 47 & & \\
& $37.3 \%$ & $62.7 \%$ & & \\
\hline
\end{tabular}

Test - Chi-square test 
Table 3: Maternal hospital stay between the SOL and IOL groups

\begin{tabular}{llcc}
\hline Hospital stay in days & Mean & SD & $\boldsymbol{p}$ value \\
\hline SOL & 2.79 & 1.4 & $<0.001$ \\
IOL & 5.85 & 2.0 & \\
\hline
\end{tabular}

Test - independent sample $\mathrm{t}$

Table 4: APGAR score for the SOL and IOL groups

\begin{tabular}{llll}
\hline APGAR score at 10 minutes & Mean & SD & $\boldsymbol{p}$ value \\
\hline SOL & 9.97 & 0.23 & 0.46 \\
IOL & 9.93 & 0.21 & \\
\hline
\end{tabular}

Test - independent sample $t$

Table 5: SCBU admission between the SOL and IOL groups

\begin{tabular}{|c|c|c|c|}
\hline SCBU admission & SOL & IOL & $p$ value \\
\hline Yes & $4 \quad(5.3 \%)$ & $2 \quad(2.6 \%)$ & 0.41 \\
\hline No & $71(94.7 \%)$ & $73(97.4 \%)$ & \\
\hline
\end{tabular}

Test - chi square test

\section{Discussion}

This study showed elective IOL at term had increased rate of cesarean section and prolonged hospital stay but no adverse perinatal and maternal outcomes.

According to the study, the rate of cesarean section was higher among women with IOL compared with SOL across all gestational ages (beyond 37 completed weeks) and parity. The NICE guideline (updated version) in July 2008 states that IOL beyond 41 weeks do not increase the rate of caesarean section compared to expectant management. The difference we observed may be due to IOL in earlier gestation associated with partially favorable cervix. A study done at Kathmandu Medical College and Teaching Hospital $(\mathrm{KMCTH})$ involving three hundred women with singleton pregnancies from 40 to 42 weeks of gestation without any risk factors showed that elective induction of labour is associated with even lesser rates of caesarean section and reduce the NICU admissions when compared with spontaneous onset of labour (14). Same findings were observed in a Canadian study conducted in 22 hospitals throughout Canada among 3,400 pregnancies at 41 or more weeks of gestation.
Another significant finding in this study was that prolonged hospital stay in IOL group compared to SOL group. This observation may be due to increased caesarean section rate in women with IOL as usual duration of hospitalisation following uncomplicated caesarean delivery is approximately three days whereas in spontaneous vaginal delivery it is 24 hours.

Our results show that there are no significant differences of adverse maternal outcomes such as duration of labour, obstetric anal sphincter injuries / genital tract trauma, and postpartum haemorrhage in the two groups. Cochrane database in 2006 reported that there was no significant difference between labour induction and control groups for postpartum haemorrhage (6). This is in line with our findings. Even better outcomes have been reported in a population based study; elective IOL from 38 weeks onwards was associated with a decreased odds of postpartum haemorrhage compared with expectant management. Induction of labour was also associated with decreased odds of anal sphincter injury compared with expectant management (14). This may be due to the large sample size involved in above study. 
Our results indicate that there are no statistically significant differences with regards to adverse perinatal outcomes such as perinatal deaths, APGAR at 10 minutes at birth $(\mathrm{p}=0.466), \mathrm{PBU} /$ NICU admission $(p=0.405)$ between the two groups. There was only one perinatal death in IOL group due to congenital heart disease. Similar results have been reported in a Cochrane database in 2006; there was no statistically significant difference in NICU admissions and APGAR scores less than 7 at 5 minutes when labour induction was compared with expectant management (6). A randomized controlled trial performed among 508 women by Heimstad et al. on IOL serial antenatal fetal monitoring in post term pregnancy has found no difference between the induced and monitored groups regarding neonatal morbidity or mode of delivery, and the outcomes were generally good (15).

\section{Conclusions and recommendations}

This study showed that elective IOL at term is associated with increased rate of cesarean section and prolonged maternal hospital stay but not with adverse perinatal and maternal outcomes when compared with SOL. Every consultant led obstetric unit should have evidence based guidelines for elective IOL for appropriate case selection. Optimum cervical ripening should be carried out prior to induction, especially for medical inductions.

\section{Acknowledgements}

We thank Dr. K.G. Imendra, Coordinator of BSc. Nursing Degree programme and all the lecturers and staff members of Allied Health Sciences Degree Programme for their support in this study. We also would like to thank Dr. Thyagi Ponnamperuma and Prof. Bilesha Perera, Department of Community Medicine for their guidance in data analysis. We pay our gratitude to the Director and all Consultants in Teaching Hospital Mahamodara, who granted permission to carry out the study in their units.

\section{References}

1. Elizabeth H, Martin MA. Anon. Oxford Concise Medical Dictionary, $8^{\text {th }}$ ed. New York. 2010; 374p.

2. Mealing NM, Roberts CL, Ford JB, Simpson JM, et al. Trends in induction of labour. New Zealand Journal of Obstetrics and Gynaecology 2009; 49(6): 599-605.
3. Sheats M, Wilcox AJ, Little RE. Post-term delivery: A challenge for an epidemiological research. Epidemioogy 1998; 9(2): 199-204.

4. Alexamder JM, Malrine DJ, Lexemo KJ. Fourty weeks and beyond. Journal of Obstetrics and Gynaecology 2000; 9(2): 219-4.

5. Balchir L, Chittaken JC, Patel RR, et al. Racial variation in the association between gestational age and perinatal mortality. British Medical Journal 2007; 334(1598): 833.

6. Gülmezoglu AM, Crowther CA, Middleton P. Induction of labour for improving birth outcomes for women at or beyond term. The Cochrane database of systematic review 2006. Available from:https://dx.doi.org/10.1002\%2F 14651858.CD004945.pub3. [Accessed on $3^{\text {rd }}$ June 2017].

7. Oslesen AW, Westergaard JG, Oslen J. Perinatal and maternal complication related to post-term delivery. American Journal of Obstetrics and Gynaecology 2003; 189(1): 222-7.

8. Crowley P. Interventions for preventing or improving the outcome of delivery at or beyond term. Cochrane Database of Systematic Reviews 1997, Issue 1. Art. No: CD000170. DOI: 10.1002/14651858.CD000170. [Accessed on 03 June 2017].

9. Induction of Labor. ACOG Practice Bulletin No. 107. American College of Obstetricians and Gynecologists Obstet Gynecol 2009; 114: 386-97.

10. Darney BG, Snowden JM, Cheng YW, at el. Elective induction of labor at term compared with expectant management: maternal and neonatal outcomes. Obstetrics and Gynaecology 2016; 122(4): 761.

11. National Institute for Health Cere Excellence. Induction of labour guideline 2008. Available from:

https://www.nice.org.uk/guidance/cg70?unlid=66220805 1201512258217. [Accessed on 03 June 2017].

12. Stewart P. Patients' attitudes to IOL. British Medical Journal 1977; (6089): 749-52.

13. Shrestha D, Bajracharya J, Shrestha NS. Maternal and neonatal outcomes of spontaneous versus induced at term. Journal of Kathmandu Medical Collage 2015. Available from: http://www.nepjol.info/index.php/JKMC/article/ view/15021. [Accessed on 03 June 2017].

14. Stock SJ, Ferguson E, Duffy A, Ford I, Chalmers J, Norman JE. Outcomes of elective induction of labour compared with expectant management. BMJ 2012; 10: 344: e2838.

15. Heimstad R, Skogvoll E, et al. Induction of labor or serial antenatal fetal. Obstet Gynecol. 2007; 109(3): 609-17. 